\title{
Therapeutic Ride: Health Service Evaluation and Satisfaction of Patients and Their Caregivers
}

\author{
Jéssica do Nascimento Pinho ${ }^{1,2}$, Edivania Anacleto Pinheiro ${ }^{2}$, Cristiane Munaretto Ferreira ${ }^{1}$, Erica Freire de \\ Vasconcelos-Pereira ${ }^{1}$, Tatiane Contin ${ }^{1}$, Vanessa Marcon de Oliveira ${ }^{1} \&$ Vanessa Terezinha Gubert de Matos ${ }^{1,2}$ \\ ${ }^{1}$ Universidade Federal de Mato Grosso do Sul. Campo Grande, Mato Grosso do Sul, Brasil \\ ${ }^{2}$ Hospital São Julião. Campo Grande, Mato Grosso do Sul, Brasil \\ Correspondence: V. T. G. Matos, Universidade Federal de Mato Grosso do Sul, Avenida Costa e Siva s/n, Cidade \\ Universitária, CEP 79070-900, Campo Grande, Mato Grosso do Sul, Brasil. Tel: 55-67-3345-7781. E-mail: \\ vanessa.matos@ufms.br
}

Received: July 2, 2020 Accepted: August 19, 2020 Online Published: September 30, 2020

doi:10.5539/gjhs.v12n12p1 URL: https://oi.org/10.5539/gjhs.v12n12p1

\begin{abstract}
Objective: To evaluate the health service and the satisfaction of patients and their caregivers in relation to the therapeutic ride, in Integrated Continuous Care. Methods: Cross-sectional, descriptive study, conducted between January 2017 and January 2018, using a specific form for data collection and interview conducted before hospital discharge. Results: The study included 23 patients and 27 caregivers. Regarding the evaluation of the service related to the therapeutic ride, $95.6 \%(22 / 23)$ of the patients and $70.4 \%(19 / 27)$ of the caregivers confirmed that they understood the objectives of this practice. The duration of the therapeutic ride was considered sufficient by $69.6 \%(16 / 23)$ of patients and $70.4 \%$ (19/27) of caregivers. Most patients $(52.2 \%)$ and caregivers $(70.4 \%)$ declared to be very satisfied with the result of the therapeutic ride. Conclusion: The therapeutic ride demonstrates its differential aspect by adding training, contributing greatly to the safe transition from the hospital to the home.
\end{abstract}

Keywords: health service research, assessment, patient satisfaction, caregivers, rehabilitation

\section{Introduction}

Worldwide, regional and national strategies, and policies are adopted for the integrated care of individuals with chronic multimorbidities in an attempt to reduce fragmented, incomplete, inefficient, and ineffective care that can lead to preventable hospitalizations (Van der Heide et al., 2015).

In response to current social and health needs, countries such as the United Kingdom, the United States, Canada, and France have instituted the integrated patient-centered care model (Leichsenring, 2004). The model implemented in Brazil is similar to the National Network of Integrated Continuous Care (RNCCI) instituted in Portugal (Lopes et al., 2010). RNCCI is defined as a set of sequential health and/or social support interventions, resulting from joint assessment, focused on recovery global, understood as the therapeutic and social support process that is active and continuous. The aim is to promote autonomy by improving the functionality of the person in a situation of dependency through his or her rehabilitation, re-adaptation, and family and social reintegration (Portugal, 2006).

In 2012, four Brazilian states (Mato Grosso do Sul, São Paulo, Paraná, and Piauí) started a pilot project called Integrated Continuous Care (ICC). This project aims to meet the new socio-health needs, characterized by the increase in individuals with loss of autonomy (Gesawordl, 2012).

Several work processes were maintained according to the Portuguese ICC model, others were adapted, and one was elaborated and implemented by the multiprofessional team of Hospital São Julião in Mato Grosso do Sul during the first hospitalizations. This new work process is called therapeutic ride and consists of the patient leaving the hospital environment exclusively for the home, within a maximum period of 48 hours, upon prior agreement among the patient, family, and care team. Throughout the hospitalization period, the patient, his or her family, and caregivers participate in the elaboration of the Singular Therapeutic Project. All of them are co-responsible for the treatment performed and are guided by the multiprofessional team on future home care. Thus, the objective of the therapeutic ride is for the patient and his or her family to perform the same hospital care during the stay at home and to observe the situations of difficulty or risk faced during the period away from the hospital to try to solve them 
with the help of the multiprofessional team (Pinho et al., 2017).

The evaluation of health services is focused on the relationship between the health need of a population and the service offered, in order to produce reliable data about its efficiency and effectiveness to improve its performance (Vieira, 2005, p.436). In this sense, Donabedian's model (Donabedian, 1988) was developed to assess the quality of care in clinical practice. Morris and Bailey (2014) add that the customer experience must also be considered when assessing the quality of services. The aim of this study was to evaluate the process and results of the therapeutic ride from the perspective of the patient and caregiver.

\section{Method}

The study was approved by the Institutional Review Board of the Federal University of Mato Grosso do Sul, in compliance with the Brazilian Resolution of the National Health Council 466/2012, and the ethical principles contained in the Helsinki Declaration.

\subsection{Design, Study Location, and Period}

This is a descriptive and quantitative study with a qualitative approach, guided by the Strengthening the Reporting of Observational studies in Epidemiology (STROBE) tool. It was developed from January 2017 to January 2018 in a ICC unit of a general, philanthropic hospital of medium complexity, located in Campo Grande, Mato Grosso do Sul, Brazil. This hospital is constituted as a rearguard unit and the ICC as a medium-term convalescence and rehabilitation unit, which has 22 beds and a specific physical space to perform this type of care.

\subsection{Population and Inclusion and Exclusion Criteria}

Patients and their caregivers admitted to the ICC who undertook at least one therapeutic ride before hospital discharge, aged 18 years and over, and who have signed the informed consent form were included in the study. Individuals who were cognitively impaired were excluded. During hospitalization, patients and caregivers were guided and trained by health professionals to perform activities that would be necessary in home care, and the therapeutic ride was proposed by consensus among the multiprofessional team, patient, and caregiver.

\subsection{Study Protocol}

The evaluation of health service quality proposes three dimensions: structure, process, and results. Structure is defined as the attributes of the settings in which care occurs. This includes the attributes of material resources (e.g., facilities, equipment, and budget), human resources (e.g., the number and qualifications of the professionals involved), and the organizational structure (e.g., the organization of the medical team, peer review methods, and refund methods) (Donabedian, 1988). Thus, the structural dimension was not considered in this study, which evaluated the process and results of the therapeutic ride.

Process was defined in this article as all the aspects involved in therapeutic ride, its definition and objectives, the duration, and the discussion about aspects of the ride after returning. As a result, the final product of the assistance provided was defined, considering contributions from the therapeutic ride and satisfaction. The data related to the process and results of the therapeutic ride were collected through a semi-structured questionnaire, applied during an interview conducted in a private room before hospital discharge.

\subsection{Analysis of Results and Statistics}

Descriptive analysis was used for sociodemographic characterization, evaluation of the work process, and results of the therapeutic ride. To evaluate continuous variables, mean, standard deviation, minimum, and maximum were calculated, and frequency and percentage were calculated for categorical variables.

\section{Results}

\subsection{Sample Characteristics}

During the study period, 30 patients underwent the therapeutic ride; however, 27 were eligible for the study. Of these, four patients were aphasic, with only their caregivers being interviewed. In total, the study included 23 patients and 27 caregivers.

The mean age observed among patients was 51.9 years ( \pm 13.60 years) and among caregivers 41.04 years $( \pm 12.36$ years). The minimum age observed among patients was 25 years and among caregivers 22 years. Regarding the bond of caregivers, 25 (92.6\%) were family members, and only two patients had a formal caregiver. The average hospital stay was 36.6 days, with a minimum of 15 days and a maximum of 60 days. The average number of therapeutic ride per patient was 1.77 , varying between one and seven. Table 1 shows the characterization of the study participants. 
Table 1. Characterization of patients and caregivers who underwent therapeutic ride during hospitalization in Integrated Continuous Care, Campo Grande, Mato Grosso do Sul, Brazil, 2017-2018

\begin{tabular}{llc}
\hline Variables & Patients (N=23) & Caregivers (N=27) \\
\hline Gender, $\mathrm{n}(\%)$ & $13(56.5)$ & $10(37.0)$ \\
Male & $10(43.5)$ & $17(63.0)$ \\
Female & & \\
\hline Age group, $\mathrm{n}(\%)$ & $5(21.8)$ & $10(37.0)$ \\
$20-39$ years & $11(47.8)$ & $14(51.9)$ \\
$40-59$ years & $7(30.4)$ & $3(11.1)$ \\
$\geq 60$ years & & \\
\hline Ethnicity, $\mathrm{n}(\%)$ & $11(47.9)$ & $18(66.7)$ \\
Brown & $9(39.1)$ & $7(25.9)$ \\
White & $3(13.0)$ & $2(7.4)$ \\
Black & & $1(3.7)$ \\
\hline Highest Level of Education Achieved, $\mathrm{n}(\%)$ & $2(8.7)$ & $10(37.0)$ \\
Whithout instruction & $11(47.9)$ & - \\
Incomplete elementary school & $3(13.0)$ & $4(14.9)$ \\
Complete elementary school & $1(4.3)$ & $6(22.2)$ \\
Incomplete high school & $4(17.4)$ & $3(11.1)$ \\
Complete high school & - & $3(11.1)$ \\
Incomplete higher education & $2(8.7)$ & \\
Complete higher education & &
\end{tabular}

During the therapeutic ride, three patients used a nasoenteral tube or gastrostomy, 13 needed to use a diaper, four needed a dressing, two were undergoing tracheostomy, three performed self-bladder catheterization, and one caregiver was responsible to perform the procedure on the patient. Regarding the use of auxiliary devices, two patients needed a simple cane, four used a cane with four supports, and two used a walker, nine used a wheelchair, and eight used a bath chair.

\subsection{Evaluation of the work process and results of the therapeutic ride}

The duration of the therapeutic ride was 48 hours, which was considered sufficient time for most patients and caregivers. The variables referring to the evaluation of the work process and the results of the therapeutic ride are shown in Table 2. 
Table 2. Evaluation of the work process and results of the therapeutic ride from the perspective of patients and caregivers, Campo Grande, Mato Grosso do Sul, Brazil, 2017-2018

\begin{tabular}{|c|c|c|}
\hline Variables & Patient $(\mathrm{N}=23)$ & $\begin{array}{c}\text { Caregivers } \\
(\mathrm{N}=27)\end{array}$ \\
\hline The definition of the therapeutic tour was explained and understood, $\mathrm{n}(\%)$ & $20(86.9 \%)$ & $22(81.5 \%)$ \\
\hline The objectives of the therapeutic ride were informed and understood, $n(\%)$ & $22(95.6 \%)$ & $19(70.4 \%)$ \\
\hline \multicolumn{3}{|l|}{ Duration of the therapeutic ride } \\
\hline Enough & $16(69.6 \%)$ & $19(70.4 \%)$ \\
\hline Insufficient (short) & $7(30.4 \%)$ & $8(29.6 \%)$ \\
\hline Discussion on aspects of the therapeutic ride on returning to the hospital, $\mathrm{n}(\%)$ & $21(91.3 \%)$ & $25(92.6 \%)$ \\
\hline \multicolumn{3}{|l|}{ Activities that you had difficulty performing during the therapeutic ride } \\
\hline Food & $2(11.1 \%)$ & $6(22.2 \%)$ \\
\hline Hugiene & $2(11.1 \%)$ & $3(11.1 \%)$ \\
\hline Medications & $1(5.5 \%)$ & $8(29.6 \%)$ \\
\hline Physical barriers & $7(38.8 \%)$ & $4(14.81 \%)$ \\
\hline Transfers and transactions at home & $4(22.2 \%)$ & $6(22.2 \%)$ \\
\hline Auxiliary device & $2(11.1 \%)$ & - \\
\hline \multicolumn{3}{|l|}{ Contribution of the therapeutic ride for health and rehabilitation, $\mathrm{n}(\%)$} \\
\hline Much & $20(87.0 \%)$ & $21(77.8 \%)$ \\
\hline Little & $3(13.0 \%)$ & $6(22.2 \%)$ \\
\hline \multicolumn{3}{|l|}{ Degree of satisfaction, $\mathrm{n}(\%)$} \\
\hline Very satisfied & $12(52.2 \%)$ & $19(70.4 \%)$ \\
\hline Satisfied & $11(47.8 \%)$ & $8(29.6 \%)$ \\
\hline Dissatisfied & - & - \\
\hline
\end{tabular}

Patients and caregivers identified the same positive results of the therapeutic ride for family living, performing routine activities, receiving visits, and improving health status. However, one patient reported the presence of severe pain, and another identified the lack of adaptation of the home to his new health condition. Caregivers identified a greater number of negative aspects of the therapeutic ride, which involved difficulty in preparing and restricting food, some failure in the preparation and administration of medicines, difficulty due to the lack of adaptation of the home, and patient with constipation, bleeding, and fall. Difficulties were accounted for by situations, not by the patient, and a patient could present more than one difficulty.

During this period, one patient left the hospital environment using a nasoenteral tube, and two patients underwent dressing at home. The enteral feeding and supplies necessary for these processes were made available by the institution; however, none of the cases reported difficulties in carrying out the procedures

\section{Discussion}

The ICC service modality is recent in Brazil. Some activities developed in this type of care are established, and others, such as the therapeutic ride, still require standardization because they are not part of the integrated care of the European or American model. The evaluation of the therapeutic ride process and its results contribute to the construction of protocols adapted to the Brazilian context and to the necessary adaptations.

The transition from care in the hospital environment to home care is a time when the family has to deal with new situations, from everyday activities to those of more complex care (Rodrigues, 2013). In general, the therapeutic ride helps patients and caregivers by reducing difficulties after discharge, and it seems to collaborate for a safe transition from the hospital to the home environment.

The presence of the caregiver during the hospitalization period provides safety to the patient, adds emotional support, and helps in the individual's physical rehabilitation during hospitalization and after discharge, in addition to assisting in adherence to treatment (Szerwieski, Cortez, \& Marcon, 2016). Most of the caregivers identified in 
this study are female, with ties of kinship or affectivity and a low level of education. The fact that most caregivers are women is related to the social role of women and the country's culture (Karsch, 2003). Reduced knowledge in health care is associated with increased care needs and a greater risk of adverse outcomes, such as hospital readmission (Samudio, Loureiro, \& Ferreira Júnior, 2016). Still, it is verified among informal care providers that the absence of a professional guiding how to perform tasks creates difficulties in the care process (Fratezi \& Gutierrez, 2011). On the other hand, health education (Siqueira, Loureiro, Frota, \& Ferreira Júnior, 2017) and training offered by the ICC health team during hospitalization seem to aid in the acquisition of knowledge about the health situation of the family and the development of skills related to care.

In the present study, most patients remained hospitalized for 30 days and underwent a therapeutic ride lasting up to 48 hours. The previous preparation through explanations about the therapeutic ride and its objectives facilitates the experience of the participants at home. In general, patients and caregivers stated that they were satisfied with the process and results provided by the therapeutic ride. Inherent in the preparation is the knowledge about what to expect during the therapeutic ride and which strategies can help in managing the situation (Meleis, Sawyer, Im, Hilfinger Messias, \& Schumacher, 2000; Santos, Carvalho, Nascimento, Sousa, \& Carvalho, 2017).

The differential aspect of the therapeutic ride is to contribute to the training and the opportunity of applying at home the knowledge acquired during the hospitalization. In the present study, the main difficulties reported by caregivers during the therapeutic ride were related to patient nutrition, transfers, and medication. Assistance in the practice of activities of daily living is a continuous task of the caregiver in the home context (Couto, Castro, \& Caldas, 2016; Vieira, Fialho, Freitas, \& Jorge, 2011) and stands out as the greatest cause of physical overload (Kodama, Spuras, \& Padula, 2009; Cabral \& Nunes, 2015).

The need for care shows the importance of training and monitoring by the team for family members and caregivers in an attempt to promote care as a lighter activity, in order to improve the quality of life of all involved. By training the family caregiver to recognize signs of worsening of the clinical condition, as well as handling the accessories and assistive devices in use, caregivers can count on more tranquility and ease during the execution of care. Thus, this training becomes a way to promote humanization with care for the caregiver and the patient, who are worn out by the situation (Vieira, Fialho, Freitas, \& Jorge, 2011; Kodama, Spuras, \& Padula, 2009).

Regarding the difficulties experienced by the patient at home, the main ones were related to the presence of physical barriers and movement at home. Similarly, the main aspects covered in the simulations by nurses to patients in rehabilitation about self-care are related to hygiene and mobility (Couto, Castro, \& Caldas, 2016).

Regarding the return to the hospital, most patients and caregivers stated that they had clarified doubts with the team and discussed the positive and negative aspects of the therapeutic ride. Such discussion enabled retraining about care and the difficulties experienced during the period of absence. When asked about the perception of learning during the therapeutic ride, patients and caregivers replied, "At home, they were able to verify risk situations and before the definitive return to the house, they cleared several doubts with the multidisciplinary team". It is important for the patient and caregivers to be heard by the multiprofessional team, when the patient and the caregiver have the opportunity to address his or her experiences and difficulties in the daily care (Inácio, Montezeli, Sade, Caveião, \& HEY, 2014; Morais, Costa, Fontes, \& Carneiro, 2009).

The professional's communication with the patient and family is an important tool, as it strengthens the adherence to oriented care and ensures the understanding of this care by the patient and his or her family (Oliveira, Quintana, Budo, Kruse, \& Beuter, 2012). Still, communication is an instrument of genuine and humanized care, since it helps in understanding the experience lived by the human being, made vulnerable by the process of illness and hospitalization (Dias et al., 2011).

Both patients and caregivers were satisfied with the therapeutic ride. The opinion of patients on the quality of services is of great importance and helps to determine which aspects of the service are useful, since patient satisfaction points to the perceived quality of the service (Javed, Liu, Mahmoudi, \& Nawaz, 2019; Mohseni, Raeisi, Azami-Aghdash, Moosavi, \& Mousavi, 2017).

Health care through home care provides some comfort and freedom, as it is the patient's own space, with unique rules for each family member. In this environment, the family and the patient seem to have greater autonomy regarding the hours of care (Oliveira, Quintana, Budo, Kruse, \& Beuter, 2012). However, part of this freedom was mentioned as a negative aspect experienced by caregivers during the therapeutic ride, as some patients did not take their medications, and some patients did not respect the medication administration schedule. Therapeutic non-adherence tends to aggravate the patient's situation (Dias et al., 2011). 


\subsection{Study Limitations}

The small number of participants is a limiting factor for consistent observations, requiring new studies to be conducted whose therapeutic ride is carried out more frequently and with approaches from the patient and caregiver in different phases of care. Due to the absence of a standardized protocol in ICC services, there is a lack of studies that discuss the strategy of the therapeutic ride with regard to family perception in relation to the duration of the walk, orientations received, care, and its applicability at home.

\section{Conclusion}

The work process involving the therapeutic ride is shown to be effective and of quality, as patients and caregivers claim to have received and understood the explanation about it and guidance from the care team regarding the necessary care during the period of absence from the hospital. Moreover, discussions about the positive and negative experiences during the therapeutic ride, between the patient and the ICC team, enabled re-training before hospital discharge.

There are well-documented benefits of a patient-centered approach that includes better access to and satisfaction with care, better recovery for discharge, reduction in costs, better health literacy and self-care, better relationships between patients and their caregivers, and a greater ability to respond to health crises (Rathert, Wyrwich, \& Boren, 2013; Bertakis \& Azari, 2011). However, there are still difficulties in the therapeutic ride, such as caregiver-centered accountability. Therefore, it is necessary to place greater emphasis to the care team on encouraging the patient's autonomy and co-responsibility.

\section{Acknowledgments}

The authors thank the Coordenação de Aperfeiçoamento de Pessoal de Nível Superior - Brasil (CAPES) and Universidade Federal de Mato Grosso do Sul (UFMS). This work was supported in part by them.

\section{Competing Interests Statement}

The authors declare that there are no competing or potential conflicts of interest.

\section{References}

Bertakis, K. D., \& Azari, R. (2011). Patient-centered care is associated with decreased health care utilization. The Journal of the American Board of Family Medicine, 24(3), 229-239. https://doi.org/10.3122/jabfm.2011.03.100170

Cabral, B. P. A. L., \& Nunes, C. M. P. (2015). Family's Caregiver Perceptions Of Care Given To The Hospitalized Aged. Revista de Terapia Ocupacional da Universidade de São Paulo, 26(1), 118-27. https://doi.org/10.11606/issn.2238-6149.v26i1p118-127. Portuguese

Couto, A. M., Castro, E. A. B., \& Caldas, C. P. (2016). Experiences To Be A Family Caregiver of Dependent Elderly In The Home Environment. Revista Rene, 17(1), 76-85. https://doi.org/10.15253/2175-6783.

Dias, A. M., Cunha, M., Santos, A. M. M., Neves, A. P. G., Pinto, A. F. C., Silva, A. S., \& Castro, S. A. (2011). Adesão ao regime Terapêutico na Doença Crônica: Revisão da Literatura. Millenium, 40(16), 201-219. Retrieved from http://revistas.rcaap.pt/millenium/article/view/8228

Donabedian, A. (1988). The Quality of CareHow Can It Be Assessed? JAMA, 260(12), 1743-1748. https://doi.org/10.1001/jama.1988.03410120089033

Fratezi, F. R., \& Gutierrez, B. A. O. (2011). Family Caregiver of Elderly Patients In Palliative Care: The Process Of Dying At Home. Ciências \& Saúde Coletiva, 16(7), 3241-3248. http://dx.doi.org/10.1590/S1413-81232011000800006 [In Portuguese]

Gesawordl. (2012). Projeto: Bases para a criação e desenvolvimento de uma Rede de Cuidados Continuados no Estado do Mato Grosso Do Sul: E2. P4 - Modelo de Cuidados Continuados e Estratégias básicas para dar impulso a criação e desenvolvimento do novo modelo [CD-ROM]. São Paulo: Gesawordl.

Inácio, L. A., Montezeli, J. H., Sade, P. M. C., Caveião, C., \& HEY, A. P. (2014). Nurse Performance In The Patient's Discharge Guidance After Kidney Transplant. Revista de Enfermagem da UFSM, 4(2), 323-331. Retrieved from http://periodicos.ufsm.br/reufsm/article/view/10186/pdf [In Portuguese]

Javed, S. A., Liu, S., Mahmoudi, A., \& Nawaz, M. (2019). Patients' satisfaction and public and private sectors' health care service quality in Pakistan: Application of grey decision analysis approaches. International Journal of Health Planning and Management, 34(1), 168-e182. https://doi.org/10.1002/hpm.2629.

Karsch, U. M. (2003). Dependent seniors: families and caregivers. Cadernos de Saúde Pública, 19(3), 861-866. 
https://doi.org/10.1590/S0102-311X2003000300019

Kodama, C. M., Spuras, M. V., \& Padula, M. P. C. (2009). Cares Given By Nurses For Rehabilitation Patients. Arquivos Médicos dos Hospitais e da Faculdade de Ciências Médicas da Santa Casa de São Paulo, 54(3), 100-6. Retrieved from http://www.fcmsantacasasp.edu.br/images/Arquivos_medicos/2009/54_3/vlm54n3_ 4.pdf. Portuguese

Leichsenring, K. (2004). Developing integrated health and social care services for older persons in Europe. International Journal Integrated Care, 4, e10. https://doi.org/10.5334/ijic.107

Lopes, M., Mendes, F., Escoval, A., Agostinho, M., Vieira, C., Vieira, I., ... Morais, C. (2010). Plano Nacional de Saúde (PNS) 2011-2016: Cuidados Continuados Integrados em Portugal - analisando o presente, perspectivando $\quad o \quad$ futuro. Évora. Retrieved from http://1nj5ms21li5hdggbe3mm7ms5.wpengine.netdna-cdn.com/files/2010/08/CSC1.pdf

Meleis, A. I., Sawyer, L. M., Im, E. O., Hilfinger Messias, D. K., \& Schumacher, K. (2000). Experiencing transitions: an emerging middle-range theory. Advances in Nursing Science, 23(1), 12-28. https://doi.org/10.1097/00012272-200009000-00006.

Mohseni, M., Raeisi, A., Azami-Aghdash, S., Moosavi, A., \& Mousavi, S. M. (2017). Hospital Services Quality from Patients' viewpoint in Iran: A Systematic Review and Meta-Analysis. Evidence Based Health Policy, Management \& Economics, 1(4), 261-9.

Morais, G. S. N., Costa, S. F. G., Fontes, W. D., \& Carneiro, A. D. (2009). Communication as a basic instrument in providing humanized nursing care for the hospitalized patient. Acta Paulista de Enfermagem, 22(3), 323-327. https://doi.org/10.1590/S0103-21002009000300014

Morris, C., \& Bailey, K. (2014). Measuring Health Care Quality: An Overview of Quality Measures. Retrieved from http://familiesusa.org/product/measuring-health-care-quality-overview-quality-measures

Oliveira, G. S., Quintana, A. M., Budo, M. L. D., Kruse, H. L., \& Beuter, M. (2012). Home Care And Hospital Assistance: Similarities And Differences From The Perspective Of The Family Caregiver. Texto \& Contexto Enfermagem, 21(3), 591-599. https://doi.org/10.1590/S0104-07072012000300014

Pinho, J. N., Monti, L. L., Santos, S. C., Siqueira, R. M. D., Paula, F. M., \& Pinheiro, E. A. (2017). The Therapeutic Walk. PECIBES, 3(2), 74-76. Retrieved from https://periodicos.ufms.br/index.php/pecibes/article/view/5265/3987 Portuguese

Portugal. (2006). Decreto Lei $n^{\circ} 101$ de 6 de Junho de 2006. Diário da República. Série - A 06 junho 2006. Retrieved

from http://www.sg.min-saude.pt/NR/rdonlyres/A110CE46-A607-4BD1-AB82-BE86B31314C3/18627/3856386 6.pdf

Rathert, C., Wyrwich, M. D., \& Boren, S. A. (2013). [Patient-centered care and outcomes: a systematic review of the literature]. Medical Care Research and Review, 70(4), 351-379. https://doi.org/10.1177/1077558712465774

Rodrigues, R. A. P., Marques, S., Kusumota, L., Santos, E. B., Fhon, J. R. S., \& Suzele, C. C. F. W. (2013). Transition of care for the elderly after cerebrovascular accidents - from hospital to the home. Revista Latino-Americana de Enfermagem, 21, 216-224. http://dx.doi.org/10.1590/S0104-11692013000700027.

Samudio, A. K. M., Loureiro, M. D. R., \& Ferreira Júnior, M. A. (2016). The Process Of Work Of Nursing Care Team Continued Integrated. Revista de enfermagem UFPE on line, 10(7), 2453-62. https://doi.org/10.5205/reuol.8423-73529-1-RV1001201607

Santos, D. F. B., Carvalho, E. B., Nascimento, M. P. S. S., Sousa, D. M., \& Carvalho, H. E. F. (2017). Elderly Care Provided By Informal Caregivers In The Home Context: Integrative Review. Sanare, 16(2), 77-84. Retrieved from https://sanare.emnuvens.com.br/sanare/article/view/1181/642

Siqueira, R. M. S., Loureiro, M. D. R., Frota, O. P., \& Ferreira Júnior, M. A. (2017). Health Education Practice In The View Of Informal Caregivers In Integrated Continuing Care. Revista de enfermagem UFPE on line, 11(8), 3079-86. https://doi.org/10.5205/reuol.11064-98681-4-ED.1108201712

Szerwieski, L. L. D., Cortez, L. E. R., \& Marcon, S. S. (2016). Caregivers of Hospitalized Adults From The Perspective Of The Nursing Staff. Revista de enfermagem UFPE on line, 10(1), 48-56. https://doi.org/10.5205/reuol.8423-73529-1-RV1001201607 
Van der Heide, I., Snoeijs S., Melchiorre, M. G., Quattrini, S., Boerma, W., Schellevis, F., Rijken, M. (2015). Innovating care for people with multiple chronic conditions in Europe. An overview. ICARE4EU. Retrieved from https://www.nivel.nl/sites/default/files/bestanden/Rapport-State-of-the-Art-ICARE4EU.pdf

Vieira, C. P. B., Fialho, A. V. M., Freitas, C. H. A., \& Jorge, M. S. B. (2011). Practices of elderly's informal caregiver at home. Revista Brasileira de Enfermagem, 64(3), 570-579. http://dx.doi.org/10.1590/S0034-71672011000300023

Vieira, E. M. (2005). Avaliação de serviços de saúde. In L. J. Franco, \& A. D. C., Passos, Fundamentos de epidemiologia (pp. 337-350, 436). Barueri: Manole.

\section{Copyrights}

Copyright for this article is retained by the author(s), with first publication rights granted to the journal.

This is an open-access article distributed under the terms and conditions of the Creative Commons Attribution license (http://creativecommons.org/licenses/by/4.0/). 